\title{
Symmetrically paired spots in the basal ganglia: a clue to fill the gap in the history of present illness of a comatose patient
}

Volume 6 Issue 5 - 2017

\section{Introduction}

Diagnostic imaging is a valuable device in clinical management of poisoned patients presented to emergency units in a comatose state. Some toxic agents have adverse effects on the central nervous system. $\mathrm{CT}$ of the brain can provide, even without contrast medium, useful information about structural disturbances of unconscious patients with suspected drug or chemical intoxication as magnetic resonance (MR) imaging does.

\section{Case report}

A 79-year-old man was found unconscious in bed by his son and transported by ground ambulance to the emergency room of our hospital one winter evening. The patient was estranged from his children, lived alone in a large garage, and drank habitually every day. On admission, the patient was in a deep coma with a Glasgow Coma Scale score of 3 (E1V1M1). His pupils were round and $2 \mathrm{~mm}$ in diameter bilaterally. They reacted briskly to light. There was no tremor, no chorea, nor increased muscle tone in extremities with normal range of joint motion. His body temperature was 36.5 degrees Celsius, his blood pressure $147 / 78 \mathrm{mmHg}$, his pulse 62 beats per minute, his respiratory rate 18 breaths per minute,and his oxygen saturation $99 \%$ while breathing ambient air. An electrocardiogram showed normal sinus rhythm, at a rate of 50 beats per minute. Inspection revealed decubitus in the back and the buttocks as well as incontinence. The arterial blood gas analysis under ambient air showed normoxia and normocapnia. His plasma glucose was $131 \mathrm{mg} / \mathrm{dL}$ (reference range, $70-110 \mathrm{mg} / \mathrm{dL}$ ), his blood level of carbon monoxide (CO) was $1.2 \%$ (< $2 \%)$, and his blood ammonium concentration was $43 \mu \mathrm{g} / \mathrm{dL}(<66 \mu \mathrm{g} /$ $\mathrm{dL})$. The remainder of his blood examination included inflammation values including $6.60 \mathrm{mg} / \mathrm{dL}$ of C-reactive protein $(<0.14 \mathrm{mg} / \mathrm{dL})$ and $6505 \mathrm{IU} / \mathrm{L}$ of creatinine phosphokinase (59-248 IU/L).

His available medical history was limited. Once his level of consciousness was determined, a careful check for hints as to the cause of the alteration in level of consciousness was undertaken. A brain computed tomographic (CT) scan without contrast medium showed bilateral hypodensity of globus pallidus, despite a diffusionweighted image (DWI) obtained 7 months before admission showing normal anatomical findings (Figure 1A \& 1B).

Because of their high metabolic activity, respiratory toxins tend to cause bilateral MR imaging abnormalities in the basal ganglia, sometimes with diffuse involvement of other brain structures. ${ }^{1}$ Carbon monoxide (CO), in particular, has a propensity to affect the globus pallidus $(\mathrm{GP}){ }^{2}{ }^{2} \mathrm{~T} 2$ prolongation in the basal ganglia is typical in acute poisoning, often with restricted diffusion on diffusion-weighted MR images. In CO poisoning, delayed leukoencephalopathy and T1 shortening in the GP may be encountered. ${ }^{2}$ When CO exposure is prolonged, cerebral endothelium undergoes oxidant stress as evident

\author{
Tatsuya Abe,' Shunsuke Ohnishi,' Takaaki \\ Ishiyama, ${ }^{2}$ Yuichiro Yoneoka ${ }^{3}$ \\ 'Department of Emergency Medicine, Niigata University Medical \\ and Dental Hospital, Japan \\ ${ }^{2}$ Department of General Medicine, Niigata University Medical \\ and Dental Hospital, Japan \\ ${ }^{3}$ Department of Neurosurgery, Niigata University Medical and \\ Dental Hospital, Japan
}

Correspondence: Yuichiro Yoneoka, Department of Neurosurgery, Uonuma Institute of Community Medicine, Niigata University Medical and Dental Hospital, 4I 32, Urasa, Minami-uonuma, Niigata, 949-7302, Japan, Tel +8I-25-777-3200, Fax+8I-25-777-5030, Email yone@bri.niigata-u.ac.jp

Received: March 24, 2017 | Published: May 08, 2017

by nitrotyrosine formation. As $\mathrm{CO}$ levels increase, modest decreases in oxygen consumption are detectable, which may reflect $\mathrm{CO}$ or nitric oxide interactions with cytochrome oxidase in regions with very low oxygen availability. ${ }^{3}$ Hemorrhagic necrosis of the putamen may be observed in cyanide and methanol poisoning, and white matter edema may be an additional feature of methanol poisoning. ${ }^{1}$

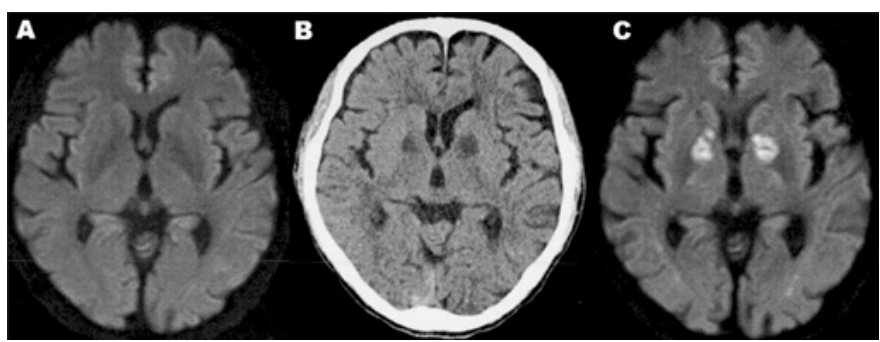

Figure I A: Axial diffusion-weighted images (DWIs) obtained 7 months before admission shows normal anatomical findings. B: Precontrast computed tomographic scan revealed bilateral hypodensity in the globus pallidus. C: DWls on the forth hospital day depict symmetric hyperintense foci in the globus pallidus.

Yarid \& Harruff ${ }^{4}$ reported that twenty-seven cases with bilateral basal ganglia lesions were identified and examined for associated or causative disease or injury with the following results: 10 cases of drug overdose, seven heart disease, three asphyxia, two chronic ethanolism, two Huntington-like disorder, and one case each of remote trauma, rheumatic heart disease, and cerebral artery gas embolism. ${ }^{4}$ Thus, this study provides no support for the assertion that globus pallidus necrosis is characteristic of $\mathrm{CO}$ poisoning. In this case, the bilateral 
GP lesions were not chronic based on normal findings of MR imaging taken 7 months before admission (Figure 1A).

We interviewed the son again and he remembered the presence of an unlit wood-burning stove in the garage where he found his father. Although the patient's blood level of $\mathrm{CO}$ was within normal range on admission, we diagnosed the patient as having suffered from $\mathrm{CO}$ poisoning through the clinical setting and the pathognomonic image finding. White-matter damage in the centrum semiovale and periventricular area and abnormalities in the globus pallidus are most commonly seen on MRI following CO exposure. ${ }^{5}$ On the forth hospital day, DWI demonstrated bilateral high signal intensity in the GP (Figure 1C), which was compatible with the diagnosis of $\mathrm{CO}$ poisoning.

\section{Authorship}

All authors had access to the data and played a role in writing this manuscript.

\section{Acknowledgements}

The authors are grateful to Hiroshi Sekiguch, M.D., Satomi Ohashi, M.D., Seigo Yamaguchi, M.D.and all staffs in the emergency room of our institute for their clinical assistance and supports as well as Katsuhiko Akiyama, M.D., and Yasuhiro Seki, M.D., for lending their expertise.

\section{Dedication}

In the memory of Shunsuke Ohnishi, M.D. You left fingerprints of grace on our hospital. You shan't be forgotten.

\section{Conflicts of interest}

None.

\section{Funding}

None.

\section{References}

1. Rachinger J, Fellner FA, Stieglbauer K, et al. MR changes after acute cyanide intoxication. AJNR Am J Neuroradiol. 2002;23(8):1398-1401.

2. O'Donnell P, Buxton PJ, Pitkin A, et al. The magnetic resonance imaging appearances of the brain in acute carbon monoxide poisoning. Clin Radiol. 2000;55(4):273-280.

3. Koehler RC, Traystman RJ. Cerebrovascular effects of carbon monoxide. Antioxid Redox Signal. 2002;4(2):279-290.

4. Yarid NA, Harruff RC. Globus Pallidus Necrosis Unrelated to Carbon Monoxide Poisoning: Retrospective Analysis of 27 Cases of Basal Ganglia Necrosis. J Forensic Sci. 2015;60(6):1484-1487.

5. Bleecker ML. Carbon monoxide intoxication. Handb Clin Neurol. 2015;131:191-203. 